\title{
ESTOQUES DE CARBONO E DIÓXIDO DE CARBONO EQUIVALENTE EM ÁRVORES DE RUA DE CIDADES BRASILEIRAS
}

\author{
CARBON AND $\mathrm{CO}_{2} E Q$ STOCKS IN STREET TREES OF BRAZILIAN CITIES
}

\author{
Allan Rodrigo Nunho dos Reis ${ }^{1}$, Daniela Biondi², Severo Ivasko Junior ${ }^{3}$, Jennifer Viezzer ${ }^{4}$, \\ Tamara Ribeiro Botelho de Carvalho Maria ${ }^{5}$, Kendra Zamproni ${ }^{6}$
}

\section{RESUMO}

As árvores urbanas proporcionam diversos benefícios para as pessoas, com destaque para a sua contribuição para a mitigação da mudança do clima por meio da fixação do carbono durante a formação da sua biomassa. Este estudo buscou estimar o estoque de carbono e dióxido de carbono equivalente ( $\mathrm{CO}_{2}$ eq) em 600 árvores de cinco espécies (Tipuana tipu, Acer negundo, Ficus benjamina, Terminalia catappa, e Licania tomentosa) plantadas na arborização viária de três cidades brasileiras (Curitiba, Paraná; Itanhaém, São Paulo; e Bonito, Mato Grosso do Sul). Para isso, foram utilizadas três equações alométricas desenvolvidas para as zonas climáticas seca e úmida, tendo o diâmetro à altura do peito e a altura total das árvores como variáveis independentes. Verificou-se que as equações para zonas secas estimaram com maior precisão o estoque de carbono, e que a espécie Tipuana tipu apresentou maior potencial para estocar carbono. Conclui-se que o desenvolvimento de equações que estimem com precisão o estoque de carbono é essencial para o conhecimento do potencial das árvores urbanas em contribuir com a mitigação dos efeitos da mudança do clima.

Palavras-chave: Equações alométricas; Floresta Urbana; Mitigação; Mudança do Clima.

\begin{abstract}
Urban trees provide a number of benefits for people, with highlights to their contribution in mitigating climate change through carbon sequestration during the formation of their biomass. This study aimed to estimate carbon and $\mathrm{CO}_{2}$ eq stocks in 600 trees of five species (Tipuana tipu, Acer negundo, Ficus benjamina, Terminalia catappa, and Licania tomentosa) planted on the street of three Brazilian cities (Curitiba, Paraná; Itanhaém, São Paulo; e Bonito, Mato Grosso do Sul). For this, we used three allometric equations developed for dry and humid climatic zones, with the diameter at breast height and the total height of the trees as independent variables. We realized that the equations for dry zones estimated the carbon stock more accurately, and that the Tipuana tipu species presented greater potential to store carbon. We concluded that the development of equations that accurately estimate the carbon stock is essential for understanding the potential of urban trees to contribute to mitigation of the effects of climate change. Keywords: Allometric equations; Climate Change; Mitigation; Urban forest.
\end{abstract}

\footnotetext{
Recebido em 17.08.2019 e aceito em 17.10.2019

1 Engenheiro Florestal. MSc. Doutorando em Engenharia Florestal, UFPR. Curitiba/PR. Email: allan.nunho@gmail.com 2 Engenheira Florestal. Dra. Professora do curso de Engenharia Florestal, UFPR. Curitiba/PR. Email: dbiondi@ufpr.br 3 Engenheiro Florestal. MSc. Doutorando em Engenharia Florestal, UFPR. Curitiba/PR. Email: severoivasko@gmail.com 4 Engenheira Florestal. MSc. Doutoranda em Engenharia Florestal, UFPR. Curitiba/PR. Email: jen.viezzer@gmail.com 5 Engenheira Florestal. MSc. Doutoranda em Engenharia Florestal, UFPR. Curitiba/PR. Email: trbotelhomaria@gmail.com 6 Engenheira Florestal. MSc. Curitiba/PR. Email: kendra.zam@gmail.com
} 


\section{INTRODUÇÃO}

A mudança do clima é atribuída às atividades humanas, como a agropecuária, o desmatamento e o consumo de combustíveis fósseis, dentre outras, que alteram a composição da atmosfera global devido ao aumento da emissão dos Gases de Efeito Estufa (GEEs), indo além da variabilidade climática natural observada em períodos de tempo comparáveis (THE INTERGOVERNMENTAL PANEL ON CLIMATE CHANGE (IPCC), 2014).

Os GEEs ocorrem naturalmente ao redor da Terra, em concentrações suficientes para manter o equilíbrio térmico do planeta e assim, as diferentes formas de vida. No entanto, os níveis crescentes desses gases, notadamente após a Revolução Industrial, contribuem para um aumento da temperatura média da atmosfera (LE QUÉRÉ et al., 2018). Em níveis pré-industriais, a concentração dos GEEs na atmosfera era de 280 ppm, enquanto em meados de 2019 esta quantidade alcançou 413,92 ppm (IPCC, 2014; NATIONAL OCEANIC AND ATMOSPHERIC ADMINISTRATION (NOAA), 2019).

Em elevadas concentrações, os GEEs são prejudiciais, pois absorvem e emitem radiação térmica ao redor da Terra, criando uma camada que impede a reflexão da radiação de volta para a atmosfera, o que promove a absorção da radiação infravermelha pela superfície do planeta e ocasiona uma mudança global no clima (RITCHIE; ROSER, 2019). Os mesmos autores afirmam que esta alteração nos padrões de temperatura promove impactos ecológicos, físicos e à saúde, incluindo eventos climáticos extremos, como enchentes, secas, tempestades e ondas de calor; elevação do nível do mar; crescimento anormal das plantações e interrupção nos sistemas hídricos.

Em nível mundial, a Organização das Nações Unidas (ONU), por meio do Painel Intergovernamental sobre Mudanças Climáticas (IPCC), orienta de forma analítica os cenários atual e futuro das mudanças no clima e os seus impactos sobre diferentes contextos. O quinto relatório elaborado pelo IPCC em 2014 organiza informações criteriosas quanto aos potenciais impactos nas mudanças do clima e as suas respectivas ações de mitigação, as quais visam a redução das fontes de emissão de GEEs e o aumento dos sumidouros que armazenam estes gases (IPCC, 2014).

Le Quéré et al. (2018) estimaram que entre 1751 e 2016, o Brasil acumulou o total de 13,88 bilhões de toneladas de dióxido de carbono $\left(\mathrm{CO}_{2}\right)$, o principal $\mathrm{GEE}$, na atmosfera. Ritchie e Roser (2019) afirmam que o Brasil é hoje a sétima economia mundial em emissões de GEEs na atmosfera, em uma tendência crescente a partir de meados do século XX.

No Brasil, diversas ações de mitigação têm sido propostas, tais como reflorestamentos, conservação de fragmentos florestais, restauração de ecossistemas, redução do desmatamento e compra de créditos no mercado de carbono (BRIANEZI et al., 2014; IPCC, 2014). No entanto, 
dependendo do local, torna-se mais complexa a definição de ações que visem à mitigação das emissões de GEEs, como é o caso das cidades, onde as opções de atuação são limitadas pelas formas e infraestruturas existentes, as quais limitam o seu potencial de adequação espacial para as ações climáticas (IPCC, 2014).

Nesse ínterim, as árvores se apresentam como elementos potenciais para compensar emissões de GEEs, devido a sua importante função na redução das concentrações destes gases na atmosfera, ao fixar $\mathrm{CO}_{2}$ durante a fotossíntese e armazená-lo como carbono em sua biomassa, tornando-se assim, um importante sumidouro de GEEs (BRIANEZI et al., 2013; NOWAK et al., 2013).

No entanto, o conhecimento sobre o potencial de armazenamento de carbono pelas árvores fora das florestas (TOFs), as quais incluem aquelas plantadas em ruas, ainda é incipiente (BRIANEZI et al., 2013; FORESTA et al., 2013; NGO; LUM, 2018; PARK et al., 2018; VIEZZER et al., 2018). Assim, torna-se necessário discutir a relevância das TOFs em estudos de armazenamento de carbono.

Desse modo, o objetivo da presente pesquisa foi estimar o estoque de carbono e de dióxido de carbono equivalente $\left(\mathrm{CO}_{2} \mathrm{eq}\right)$ em indivíduos das cinco espécies arbóreas mais frequentes nas ruas de três cidades brasileiras, e analisar a sua contribuição para a mitigação da mudança do clima.

\section{MATERIAL E MÉTODOS}

\section{Áreas de estudo}

As áreas de estudo incluem as cidades brasileiras de Curitiba, capital do Estado do Paraná (PR); Itanhaém, no litoral de São Paulo (SP); e Bonito, localizada no Mato Grosso do Sul (MS).

Curitiba localiza-se no bioma Mata Atlântica. O marco zero da cidade situa-se a $25^{\circ} 25^{\prime} 46,89^{\prime \prime} S$ e 49¹6'16,56" W, com 435,27 km² de área total, dos quais 94,6\% são urbanizados (INSTITUTO BRASILEIRO DE GEOGRAFIA E ESTATÍSTICA (IBGE), 2016).

O município de Itanhaém também está no bioma Mata Atlântica, tem seu marco zero situado à 2411"1' S e 4647"22' W (ITANHAÉM, 2019) e uma extensão territorial de 601,7 km², com área urbanizada de 6,8\% (IBGE, 2016).

Já o município de Bonito localiza-se no Cerrado e tem seu núcleo urbano sob as

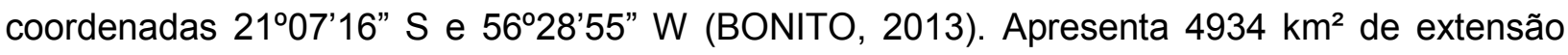
territorial, dos quais menos de $1 \%\left(3,48 \mathrm{~km}^{2}\right)$ compreendem a área urbanizada (IBGE, 2016). 
Segundo a classificação de Köppen-Geiger, o clima no município de Curitiba é do tipo $\mathrm{Cfb}$, com temperatura média anual de $17^{\circ} \mathrm{C}$ e precipitação média anual de $1400 \mathrm{~mm}$; em Itanhaém o clima é do tipo Af, com precipitação média de 2000 mm, caracterizado como clima tropical sem estação seca caracterizado como clima tropical sem estação seca, com temperatura média anual de $25^{\circ} \mathrm{C}$; o clima de Bonito é do tipo Aw, com $1200 \mathrm{~mm}$ de média mensal de precipitação e $23,1 \stackrel{\circ}{ } \mathrm{C}$ de temperatura média anual, apresentando uma estação seca acentuada entre junho e setembro e precipitações mais frequentes de novembro a janeiro (ALVAREZ et al., 2014).

Curitiba possui aproximadamente 320.000 árvores em vias públicas (CURITIBA, 2018), estando Acer negundo L. (acer) e Tipuana tipu (Benth.) Kuntze (tipuana) entre as principais espécies plantadas, as quais representam 4,68 e 6,35\% do total de árvores nas ruas, respectivamente (BOBROWSKI; BIONDI, 2012).

A área urbana de Itanhaém possui aproximadamente 18.128 indivíduos vegetais de porte arbóreo nas ruas, com maior frequência de árvores da espécie Terminalia catappa L. (setecopas, chapéu-de-sol) (17,5\%) e Ficus benjamina L. (figueira-benjamim) (16,8\%) (MARIA; BIONDI; BOBROWSKI, 2016).

Em Bonito, a arborização viária conta com 6.524 indivíduos de porte arbóreo, sendo composta principalmente por Licania tomentosa (Benth.) Fritsch. (oiti), que representa 63,3\% da arborização (ZAMPRONI; BIONDI; BOBROWSKI, 2016).

\section{Procedimentos metodológicos}

Nos inventários florestais urbanos realizados por Bobrowski e Biondi (2012) em CuritibaPR; Maria, Biondi e Bobrowski (2016) em Itanhaém-SP e Zamproni, Biondi e Bobrowski (2016) em Bonito-MS, foram utilizadas dentre outras variáveis, o Diâmetro à Altura do Peito (DAP) e altura total dos indivíduos arbóreos presentes nas ruas, as quais foram empregados para quantificar o carbono.

Foram utilizadas três equações alométricas para determinar a quantidade de carbono presente nas cinco espécies estudadas: Acer negundo e Tipuana tipu (Curitiba), Ficus benjamina e Terminalia catappa (Itanhaém), e Licania tomentosa (Bonito). Estas espécies foram selecionadas por serem as mais plantadas nas três cidades, de acordo com pesquisas de campo realizadas anteriormente. Para cada espécie foram amostrados 120 indivíduos, totalizando 600 árvores amostradas nas três cidades.

As equações A e B (Quadro 1) foram desenvolvidas por Brown (1997) e dependem do regime de precipitação anual de cada município. $A$ equação $A$ foi utilizada para determinar a 
quantidade de carbono de árvores tropicais em zona climática seca (com precipitação anual entre 900 e 1500 mm), correspondente às regiões de Curitiba e Bonito, enquanto a equação B aplicase às árvores presentes na zona climática úmida (cuja precipitação anual varia entre 1500 e 4000 $\mathrm{mm}$ ), característica correspondente à região de Itanhaém.

Essas equações, que determinam originalmente a quantidade de biomassa das árvores, foram adaptadas para expressar a variável de interesse da pesquisa, no caso, a quantidade de carbono presente nas árvores. Para isso, o resultado em $\mathrm{kg}$ encontrado foi multiplicado por 0,47, uma vez que a biomassa de árvores folhosas possui $47 \%$ de carbono em média, e em seguida este valor foi dividido por 1000 para a expressão da quantidade de carbono em toneladas (IPCC, 2014).

A equação C (Quadro 1) foi desenvolvida por Brianezi et al. (2013) para árvores de rua em Viçosa, Minas Gerais, com base no modelo proposto por Schumacher e Hall (1933), embora também estime a quantidade de carbono presente nas árvores, tem aplicação independente do regime de precipitação da região (Equação C) e, desta forma, foi utilizada para as cinco espécies avaliadas.

Para a conversão em dióxido de carbono equivalente $\left(\mathrm{CO}_{2} \mathrm{eq}\right)$, a quantidade de carbono encontrada nas três equações foi multiplicada por 3,67, conforme recomendado por IPCC (2014).

\begin{tabular}{|c|c|}
\hline Equação & Fórmula \\
\hline $\mathrm{A}$ & $t C=\frac{[\exp (-1,996+2,32 * \ln D A P)] * 0,47}{1000}$ \\
\hline $\mathrm{B}$ & $t C=\frac{\left[42,69-(12,800 * D A P)+\left(1,242 * D A P^{2}\right)\right] * 0,47}{1000}$ \\
\hline $\mathrm{C}$ & $t C=\frac{\exp [-0,906586+(1,60421 * \ln D A P)+(0,37162 * \ln h)]}{1000}$ \\
\hline
\end{tabular}

Nota: $\mathrm{tC}=$ quantidade de carbono (toneladas); DAP = diâmetro à altura do peito $(\mathrm{cm}) ; \mathrm{h}=$ altura total $(\mathrm{m})$.

Quadro 1. Equações alométricas utilizadas para o cálculo do carbono e $\mathrm{CO}_{2} \mathrm{eq}$.

Chart 1. Allometric equations used for carbon and $\mathrm{CO}_{2} \mathrm{eq}$ calculation.

Além disso, de acordo com Nowak (1994), as árvores de rua apresentam aproximadamente $80 \%$ da biomassa daquelas localizadas em florestas naturais. Assim, os resultados obtidos para as árvores amostradas foram multiplicados por 0,8 , visando deixá-los mais coerentes com o contexto urbano. 


\section{RESULTADOS E DISCUSSÃO}

A quantidade de carbono e $\mathrm{CO}_{2}$ eq, em toneladas, armazenados pelos indivíduos de $A$. negundo, T. tipu e L. tomentosa amostrados é apresentada na Tabela 1. Por possuírem maior DAP e altura total, os indivíduos de T. tipu apresentaram mais toneladas de carbono e $\mathrm{CO}_{2} \mathrm{eq}$ armazenados do que $A$. negundo e L. tomentosa juntas, uma vez que os indivíduos de $T$. tipu apresentaram DAP e altura média de $61,43 \mathrm{~cm}$ e $19 \mathrm{~m}$, respectivamente, enquanto para $A$. negundo as médias de DAP foram de $31,08 \mathrm{~cm}$ e de altura de $8 \mathrm{~m}$, e para $L$. tomentosa, as médias foram 20,17 cm de DAP e 6,16 m de altura.

Tabela 1. Estoque de carbono e $\mathrm{CO}_{2}$ eq nas árvores localizadas em regiões de zona seca Table 1. Stock of carbon and CO2eq in trees located in dry zone regions

\begin{tabular}{|c|c|c|c|c|c|c|}
\hline \multirow{2}{*}{ Espécie } & \multirow{2}{*}{$\mathbf{n}$} & \multirow{2}{*}{ Local } & \multicolumn{2}{|c|}{ (Eq. A ou B) Brown (1997) } & \multicolumn{2}{|c|}{ (Eq. C) Brianezi et al. (2013) } \\
\hline & & & tC & tCO2eq & tC & tCO2eq \\
\hline $\begin{array}{c}\text { Acer } \\
\text { negundo }\end{array}$ & 120 & Curitiba/PR & 20,6102 & 75,6396 & 21,5821 & 79,2062 \\
\hline $\begin{array}{c}\text { Tipuana } \\
\text { tipu }\end{array}$ & 120 & Curitiba/PR & 95,1689 & 349,2701 & 88,5090 & 324,8279 \\
\hline $\begin{array}{l}\text { Licania } \\
\text { tomentosa }\end{array}$ & 120 & Bonito/MS & 9,6378 & 35,3711 & 11,1411 & 40,8880 \\
\hline $\begin{array}{c}\text { Terminalia } \\
\text { catappa }\end{array}$ & 120 & Itanhaém/SP & 32,0289 & 117,5462 & 18,1837 & 66,7342 \\
\hline $\begin{array}{c}\text { Ficus } \\
\text { benjamina }\end{array}$ & 120 & Itanhaém/SP & 16,2438 & 59,6146 & 9,4283 & 34,6017 \\
\hline TOTAL & 600 & & 173,6896 & 637,4416 & 148,8442 & 546,2580 \\
\hline
\end{tabular}

Nota: $\mathrm{n}=$ número de indivíduos amostrados; $\mathrm{tC}$ = toneladas de carbono; $\mathrm{tCO}_{2} \mathrm{eq}=$ toneladas de dióxido de carbono equivalente.

Castro Neto e Sarmento (2019), em estudo realizado com árvores de rua de Lisboa, em Portugal, também encontraram valores superiores de estoque de carbono pelas árvores da espécie T. tipu, com um total de 403 tC estimados para 1070 indivíduos, em relação às da espécie $A$. negundo, com um total de 98 tC estimados para 1241 árvores da espécie.

Foi possível observar que os resultados obtidos com a aplicação da equação para clima seco e a equação geral foram relativamente próximos, sugerindo que estas equações são boas indicadoras do potencial de armazenamento de carbono para árvores urbanas.

As árvores da zona úmida amostradas ( $T$. catappa e $F$. benjamina) obtiveram quantidades inferiores de carbono armazenado em relação às da zona seca. No entanto, estes resultados divergem da maioria dos trabalhos relacionados às florestas plantadas para fins comerciais, onde aquelas localizadas em zonas úmidas tendem a apresentar um estoque maior 
de carbono em relação às zonas secas (KURUPPUARACHCHI; SENEVIRATHNE, 2011; PEREIRA JÚNIOR et al., 2016).

Além disso, foi possível verificar que as árvores de T. catappa podem estocar juntas, aproximadamente o dobro de $\mathrm{tC}$ e $\mathrm{CO}_{2}$ eq que os indivíduos de F. benjamina, e que esta distinção se deve principalmente à diferença de altura entre as espécies. Os indivíduos de T. catappa apresentaram altura média de 6,78 $\mathrm{m}$, enquanto para os da espécie $F$. benjamina a altura média foi de 3,92 m, apesar dos DAPs médios terem sido relativamente próximos, com 27,44 cm para T. catappa e $19,19 \mathrm{~cm}$ para $F$. benjamina.

A equação para clima úmido superestimou em $74 \%$ a quantidade de $\mathrm{CO}_{2}$ eq estocado em relação à equação que não considera a zonas climáticas em que as árvores estão localizadas (Equação C) e, desta forma, não se recomenda a utilização da equação desenvolvida para zonas climáticas úmidas.

Os resultados encontrados mostram a importância das árvores de rua na mitigação da mudança do clima devido a sua produção de biomassa e, consequentemente, fixação de $\mathrm{C}$ e remoção de $\mathrm{CO}_{2}$ eq da atmosfera. Estes resultados podem ser comparados às emissões de GEEs provenientes de veículos, conforme Velasco e Higuchi (2009), que realizaram uma estimativa do carbono fixado por 920 árvores de diferentes espécies plantadas na cidade de São Paulo, SP, e compararam os resultados com a emissão média de $\mathrm{CO}_{2}$ por veículos leves. Os autores constataram que as árvores estocavam aproximadamente 145,26 tC ha-1 e que um hectare de vegetação poderia mitigar a emissão de 20 veículos leves.

Estudos têm confirmado que os estoques de carbono em árvores urbanas diferem dos encontrados em florestas nativas, conforme assinalado por Nowak (1994), Brianezi et al. (2013) e Nowak et al. (2013). Isto pode ser atribuído pela densidade de árvores em um local, às distribuições de tamanho das árvores, à composição de espécies e à arquitetura da copa (NOWAK et al., 2013; NGO; LUM, 2018).

De modo geral, as árvores fora das florestas têm maior área para se desenvolver e, desta forma, produzem mais biomassa e estocam mais carbono por indivíduo do que árvores em uma floresta (BROWN, 1997; BRIANEZI et al., 2013), considerando que não sofram com manejo que afete a sua arquitetura natural e limitem o seu desenvolvimento (NOWAK et al., 2013).

Por outro lado, segundo Ngo e Lum (2018), as equações ajustadas a partir de florestas nativas podem superestimar sistematicamente as quantidades de carbono estocado em árvores de rua, porque com o mesmo DAP, as árvores das florestas tendem a ser mais altas. Os autores explicam que isto se deve às árvores em florestas precisarem alocar recursos para o crescimento em altura e só começarem a ramificar próximo ao meristema apical.

Devido a essas incertezas quanto ao potencial de fixação de carbono por árvores de rua e à carência de equações alométricas ajustadas para o contexto urbano, somado à impossibilidade 
de acesso a dados dendrométricos de todas as espécies plantadas nas três cidades estudadas, no presente trabalho não foi possível realizar uma aplicação mais ampla dos resultados. Ainda assim, os resultados encontrados para árvores isoladas podem ser comparados aos de árvores presentes em fragmentos de vegetação. Viezzer et al. (2018) estimaram o estoque de carbono e carbono equivalente em indivíduos das espécies $A$. negundo e T. tipu nas ruas de Curitiba e verificaram que, juntas, as árvores destas espécies podem estocar quantidades de carbono comparáveis aos constituídos por fragmentos florestais urbanos.

Desse modo, ainda que tenham sido amostrados indivíduos arbóreos que não representam completamente a arborização de suas respectivas cidades, pelo fato de estarem entre as espécies mais plantadas em cada local, os resultados deste estudo demonstram a importância de se considerar o armazenamento de carbono em árvores de rua para a elaboração de políticas que visem o desenvolvimento de cidades mais sustentáveis.

\section{CONCLUSÕES}

Em geral, as árvores avaliadas de clima seco estocaram maior quantidade de carbono do que as de clima úmido, com destaque para a espécie Tipuana tipu.

Considerando que a equação para clima úmido superestimou a quantidade de $\mathrm{CO}_{2} \mathrm{eq}$ estocado em relação à equação geral, recomenda-se utilizar a equação $\mathrm{C}$ para estimar o estoque de carbono em árvores urbanas localizadas em áreas úmidas.

Em pesquisas para estimativa do estoque de carbono em árvores de clima seco, sugerese a utilização da equação específica para este contexto, devido ao fato dos resultados encontrados terem sido próximos ao da equação geral. Além disso, devem ser desenvolvidas equações que permitam estimar com maior precisão a quantidade de carbono estocado nas árvores urbanas, fornecendo subsídios a ações de mitigação das mudanças do clima por meio destas árvores.

\section{AGRADECIMENTOS}

À Coordenação de Aperfeiçoamento de Pessoal de Nível Superior (CAPES) pela concessão das bolsas para a realização do estudo. 


\section{REFERÊNCIAS}

ALVAREZ, C. A.; STAPE, J. L.; SENTELHAS, P. C.; GONÇALVES, J. L. M.; SPAROVEK, G. Köppen's climate classification map for Brazil. Meteorologische Zeitschrift, Stuttgart, v. 22, n. 6, p. 711-728, 2014.

BONITO. Prefeitura Municipal. História. 2013. Disponível em: <http://www.bonito.ms.gov.br/ bonito/historia>. Acesso em: 02 jul. 2019.

BOBROWSKI, R.; BIONDI, D. Caracterização do padrão de plantio adotado na arborização de ruas de Curitiba, Paraná. Revista da Sociedade Brasileira de Arborização Urbana, v. 7, n. 3, p. 20-30, 2012.

BRIANEZI, D.; JACOVINE, L. A. G.; SOARES, C. P. B.; CASTRO, R. V.; BASSO, V. M. Equações alométricas para estimativa de carbono em árvores de uma área urbana em ViçosaMG. Árvore, Viçosa, v. 37, n. 6, 2013.

BRIANEZI, D.; JACOVINE, L. A. G.; SOARES, C. P. B.; GONÇALVES, W.; ROCHA, S. J. S. S. Balanço de emissões e remoções de Gases de Efeito Estufa no campus da Universidade Federal de Viçosa. Floresta e Ambiente, Seropédica, v. 21, n. 2, 2014.

BROWN, S. Estimating biomass and biomass change of tropical forests: a primer. Rome: FAO, 1997.

CASTRO NETO, M.; SARMENTO, P. Assessing Lisbon trees' carbon storage quantity, density, and value using open data and allometric equations. Information, Basel, v. 10, n. 4, p. 1-16, 2019.

CURITIBA. Conheça as árvores que compõem a paisagem de Curitiba. 2018. Disponível: $<$ https://www.curitiba.pr.gov.br/noticias/conheca-as-arvores-que-compoem-a-paisagem-decuritiba/45657>. Acesso em 16 ago. 2019.

FORESTA, H.; SOMARRIBA, E.; TEMU, A.; BOULANGER, D.; FEUILLY, H.; GAUTHIER, M. Towards the assessment of trees outside forests: resources assessment working. Paper 183. FAO Rome. 2013.

INSTITUTO BRASILEIRO DE GEOGRAFIA E ESTATÍSTICA (IBGE). Censo. 2010.

Disponível em: <http://censo2010.ibge.gov.br/noticiascenso?view=noticia\&id=3\&idnoticia=1766 \&busca=1\&t=censo-2010-populacao-brasil-190-732-694-pessoas>. Acesso em: 20 mai. 2019.

ITANHAÉM. Prefeitura municipal. 2019. Disponível em: <http://www2.itanhaem.sp.gov.br/ turismo/dados-gerais/>. Acesso em: 02 jul. 2019.

KURUPPUARACHCHI, K. A. J. M.; SENEVIRATHNE, G. Carbon stocks in selected dry and wet zone forests of Sri Lanka. In: PROCEEDINGS OF INTERNATIONAL FORESTRY AND ENVIRONMENT SYMPOSIUM, 2011, Colombo. Anais... Colombo: ICIMOD, 2011. Disponível em: <http://journals.sjp.ac.lk/index.php/fesympo/article/view/108>. Acesso em: 05 jul. 2019.

LE QUÉRÉ, C.; ANDREW, R. M.; FRIEDLINGSTEIN, P.; SITCH, S.; PONGRATZ, J.; MANNING, A. C. et al. Global Carbon Budget 2017. Earth System Science Data, Geneva, v. 10, 405-448, 2018. doi: https://doi.org/10.5194/essd-10-405-2018 
MARIA, T. R. B. C.; BIONDI, D.; BOBROWSKI, R. Inventário florístico quali-quantitativo das vias públicas de Itanhaém-SP. Revista da Sociedade Brasileira de Arborização Urbana. Piracicaba, v. 11, n. 4, p. 79-97, 2016.

NGO, K. M.; LUM, S. Aboveground biomass estimation of tropical street trees. Journal of Urban Ecology, Oxford, v. 4, n. 1, 2018.

NATIONAL OCEANIC AND ATMOSPHERIC ADMINISTRATION (NOAA). Trends in Atmospheric Carbon Dioxide. 2019. Disponível em: <https://www.esrl.noaa.gov/gmd/ ccgg/trends/>. Acesso em: 16 jul. 2019.

NOWAK, D. J. Atmospheric carbon dioxide reduction by Chicago's urban forest. In: MCPHERSON, E. G.; NOWAK, D. J.; ROWNTREE, R. A. (Eds.). Chicago's Urban Forest Ecosystem: results of the Chicago Urban Forest Climate Project. USDA Forest Service General Technical Report NE-186, Radnor, p. 83-94. 1994.

NOWAK, D. J.; GREENFIELD, E. J.; HOEHN, R. E.; LAPOINT, E. Carbon storage and sequestration by trees in urban and community areas of the United States. Environmental Pollution, Amsterdam, v. 178, p. 229-236, 2013.

PARK, J. H.; BAEK, S. G.; KWON, M. Y.; JE, S. M.; WOO, S. Y. Volumetric equation development and carbon storage estimation of urban forest in Daejeon, Korea. Forest Science and Technology, Londres, v. 14, n. 2, p. 97-104, 2018.

PEREIRA JÚNIOR, L. R.; ANDRADE, E. M.; PALÁCIO, H. A. D.; RAYMER, P. C. L.; RIBEIRO FILHO, J. C.; PEREIRA, F. J. S. Carbon stocks in a tropical dry forest in Brazil. Revista Ciência Agronômica, Fortaleza, v. 47, n. 1, p. 32-40, 2016.

RITCHIE, H.; ROSER, M. $\mathbf{C O}_{2}$ and other Greenhouse Gas Emissions. 2019. Disponível em: $<$ https://ourworldindata.org/co2-and-other-greenhouse-gas-emissions>. Acesso em: 07 mai. 2019.

THE INTERGOVERNMENTAL PANEL ON CLIMATE CHANGE (IPCC). Climate change 2014: mitigation of climate change - Contribution of Working Group III to the Fifth Assessment Report of the Intergovernmental Panel on Climate Change. New York: Cambridge University Press. 2014. $1454 \mathrm{p}$.

VELASCO, G. D. N.; HIGUCHI, N. Estimativa de sequestro de carbono em mata ciliar: Projeto POMAR, São Paulo (SP). Ambiência, Guarapuava, v. 5, n. 1, p. 135-141, 2009.

VIEZZER, J.; BIONDI, D.; REIS, A. R. N.; SILVA, D. A.; MORAES, E. N. Estoques de carbono e CO2eq em Acer negundo e Tipuana tipu na arborização de ruas de Curitiba, Paraná. In: XXII CONGRESSO BRASILEIRO DE ARBORIZAÇÃO URBANA, 2018, Salvador. Anais... Salvador: SBAU, 2018. p. 54-57.

ZAMPRONI, K.; BIONDI, D.; BOBROWSKI, R. Avaliação quali-quantitativa da espécie Licania tomentosa (Benth.) Fritsch. na arborização viária de Bonito-MS. Revista da Sociedade

Brasileira de Arborização Urbana, v. 11, n. 2, p. 45-58, 2016. 